\title{
PERFORMANCE OF DIGITAL VIDEO BROADCASTING- TERRESTRIAL (DVB-T) USING OFDM AS SYSTEM
}

\author{
Drakshayini M N ${ }^{1}$, Arun Vikas Singh ${ }^{2}$, Vyshanava Nandini $S^{3}$ \\ ${ }^{1}$ Associate Professor, Electronics and Communication Department, TJIT, Karnataka, India \\ ${ }^{2}$ Head of the Department, ECE, TJIT, Karnataka, India \\ ${ }^{3} M$-tech student, Electronics and Communication Department, TJIT, Karnataka, India
}

\begin{abstract}
In this paper the Performance of Digital Video Broadcasting-Terresrial(DVB-T) Transmission and reception part is undertaken.Any audio,video or image is taken as input and calculating the Bit Error Rate(BER) between transmitted and received image then we are analysing the performance of the system for different Signal To Noise Ratio(SNR) values. The system is analysed for different types of channels such as AWGN, Rayleigh and Rician channels. Finally a comparative study of the systems for different channel and SNR conditions is analysed. DVB-T is a system that makes use of the Orthogonal Frequency Division Multiplexing(OFDM) concept for the faster transmission and reception of the data. It also involves the error detection and correction techniques by the forward error correction techniques.
\end{abstract}

Keywords: DVB-T,OFDM, Inverse And Fast Fourier Transform,Quadrature Amplitude Modulation.

\section{INTRODUCTION:}

Frequency-DivisionMultiplexing (FDM) is a system in which many signals are combined for transmission on a only communications line or station. It is an Analog technique. Each signal is assigned a different frequency within the main channel as shown in the Figure1. However the communication channel must have a very large bandwidth which is major drawback. An alternative to this technique is the Orthogonal frequency-division multiplexing (OFDM) which overcomes the above mentioned drawback.

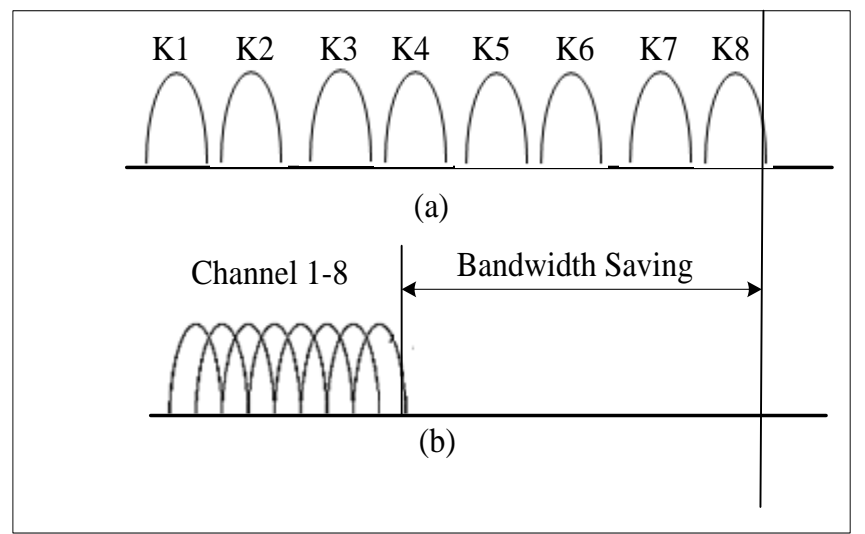

Figure1: a.Frequency Division Multiplexing (FDM) b. Orthogonal Frequency Division Multiplexing (OFDM).

\subsection{OFDM}

It is a special kind of modulation technique for applications such as DAB and DVB systems.OFDM is a type of multichannel modulation that divides a given channel into parallel sub channels so that multiple symbols are sent in parallel. DVB-T is a digital transmission system that delivers a series of data at symbol rate. Digital Video
Broadcast- Terrestrial system transmits compressed digital audio, digital video and other data in an MPEG stream, using OFDM. A key advantage of using OFDM is that multi carrier modulation can be implemented in the discrete domain byusing IDFT or computationally efficient FFT. The use of OFDM helps the receiver to counter the effects of multipath in urban environment. In DVB-T there are two choices for the number of carriers, one is known as $2 \mathrm{~K}$ mode which is of 1,705 carriers. $8 \mathrm{~K}$ mode which consists of 6,817 carriers.Effects of multipath could also be avoided using the guard interval bit insertion. By choosing an appropriate length of the guard interval.

\subsection{Ofdm Modulation and Demodulation:}

Inverse Fast Fourier Transformation(IFFT) is used in modulation technique and Fast Fourier Transformation(FFT) is used in demodulation technique. At the receiver of the DVB-T receiver end, the OFDM demodulation is performed which is achieved by using Fast Fourier Transformation.

\section{PROPOSED SYSTEM:}

In this section Source coding and MPEG2 is a Standard container format for transmission and storage of audio, video and programs and Programme stream PS is just a container format for multiplexing audio, video and data.Transport stream $\mathrm{TS}$ is a Container format for transmission as well as storage of audio, video and data. Example movie, news cart, sports news which displays on TV.Encoder block: It is the first level of protection applied to transmitted data. Vulnerable and strong in bursting the errors. This block is able to detect and correct multiple symbol errors. RS code is one of the linear block code. 
(n,k,n-k+1)is for constructing the encoder object we use Forward error correcting (FEC) is used for controlling errors in data. Second section isconvolution encoder: It is specially for error detection and error correction. This block encrypts a sequence of data to produce a sequence of binary output paths.Converts decimal form into binary form. This block can process multiple symbols at a time. To specify the encoder we use trellis structure parameter. Interleaver block in this section Convolution interleaver is used to reorder the spreaded data.Improves error rate in communication system whose channel produces errors.Data type of this output will be the same as that of the input signal. It Consists of set of shift register each with fixed delay.block interleaver: Data sequence is rearranged again also called as random interleaver.

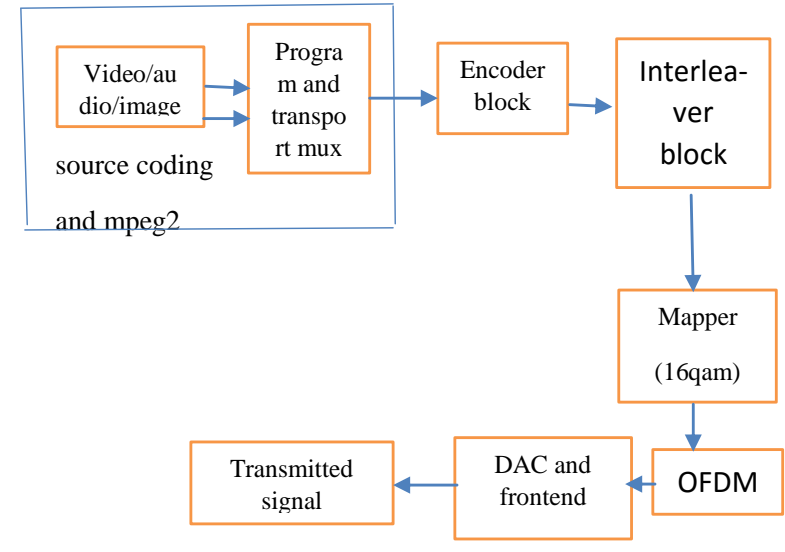

Figure2: Block diagram of digital video broadcasting(DVB) Terrestrial

Mapper maps the bits to complex values (I/Q). Complex moderated values are called symbols. Set of symbols are called constellation. QAM modulator: QAM encodes information in both ASK and PSK.We are using 16qam here 16 refers to number of points on constellation, it is 4 bits per symbol(1/4 bit rate).Existing system uses QAM modulation so 16 constellation points are used. To have unlike constellation values, data is divided in groups of 4 bits each and convert that binary code to grey code for better accuracy. Higher two bits are used for unreal number and lesser two bits are used to signify real number.

OFDM in this section, FFT and IFFT are used in to multiplex the signal together at the transmitter and demultiplex the signal in the receiver.FFT/IFFT must be able to carry $2 \mathrm{~K} / 8 \mathrm{~K}$ points in carrier spacing interval. Guard interval is used to eliminate intersymbol interference.DAC is a function that converts digital data into an Analog signal.ADC is a device that converts a continuous signal to digital data. Data rate of $6 \mathrm{Mbps}$ to $48 \mathrm{Mbps}$ is maintained. An OFDM signal, which is produced at the transmitter end by using DVB-T $2 \mathrm{k}$ mode carrier and transmitted over noisy communication stations i.e. AWGN, Rayleigh and Rician. At the 16 QAM modulation scheme the output will be observed at the value of SNR and BER is evaluated for the performance of OFDM signal.

\section{FLOW OF THE PROJECT:}

We intend to calculate the Bit Error rate between the transmitted and received image and tabulate the performance of the system for different SNR and Channel conditions and plot the performance.

1. Read the image as the input data.

2. Apply the First level of encoder.

3. Apply the First level of interleaver.

4. Apply the Second level of encoder.

5. Apply the Second level of interleaver.

6. Apply the OFDM modulation.

7. Pass the Transmitted data through the channel.

8. Apply the OFDM demodulation.

9. Apply the Second level of de-interleaver

10. Apply the Second level of decoder.

11. Apply the First level of de-interleaver.

12. Apply the First level of decoder.

13. Receive the image.

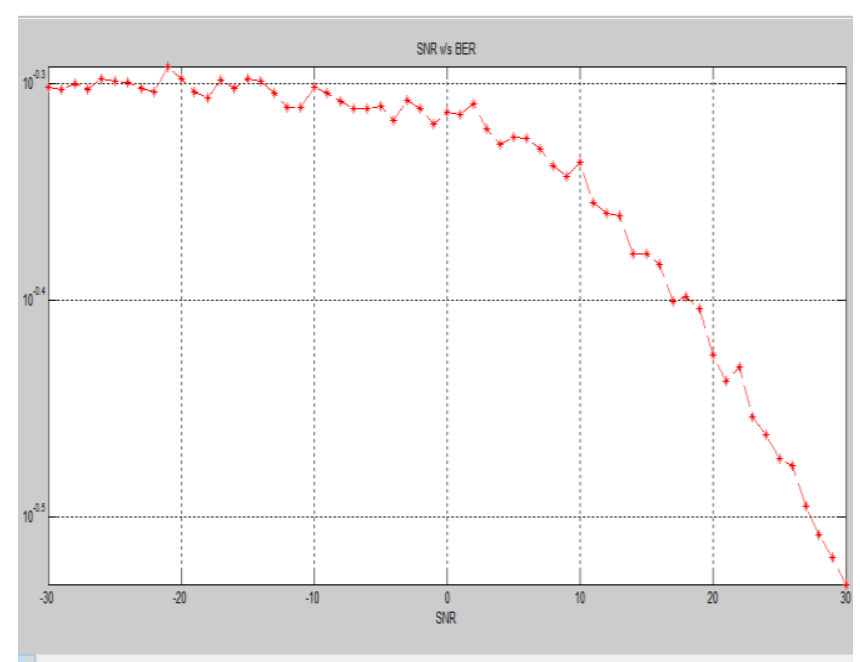

Figure3: Graph shows SNR/BER for OFDM system

\section{RESULT EVALUATION:}

Bit Error Rate(BER) is the number of bit errors per unit time. The bit error ratio is the number of bit errors divided by the total number of transferred bits during a studied time interval.

Signal to Noise Ratio(SNR) is defined as the ratio of signal power to the noise power, often expressed in decibels. Below figure shows recovered input for different SNR values from $-30 \mathrm{Db}$ to $+30 \mathrm{Db}$ 


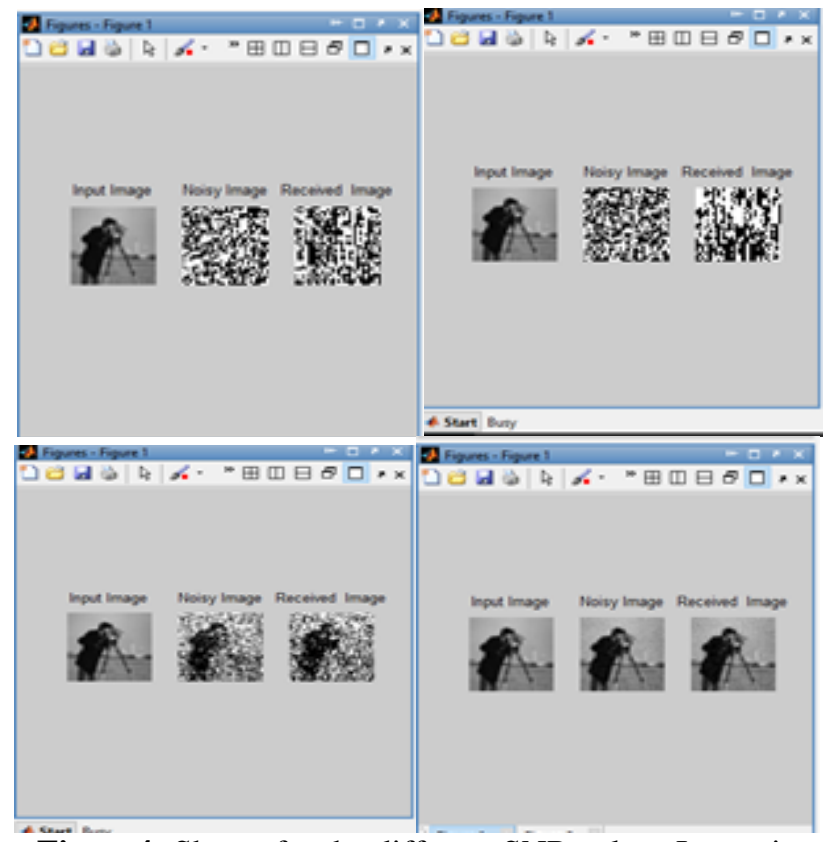

Figure4: Shows for the different SNR values Image is recovered.

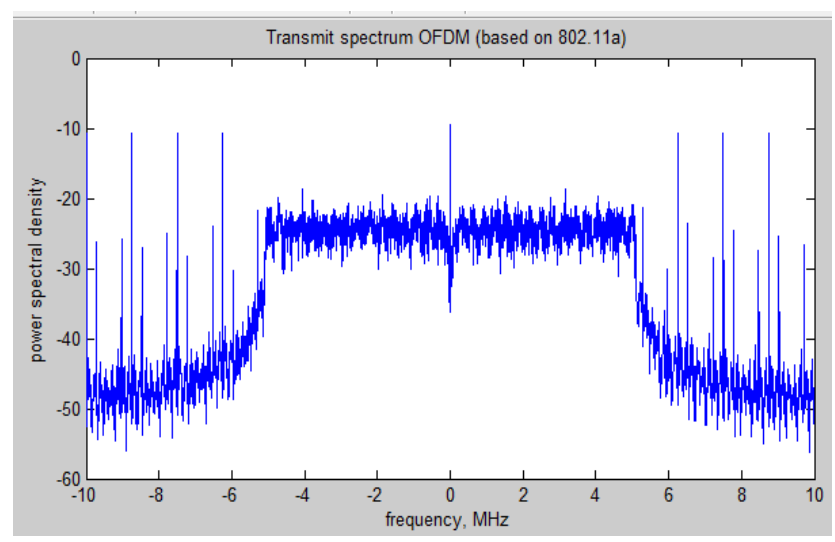

Figure4: shows the normalized Power spectrum graph of OFDM using welch method power spectral density.

\section{CONCLUSION}

Transmission and reception was demonstrated with there weltch power spectral density.normalized graph of OFDM is noted and the parameters used here are depend on $2 \mathrm{k}$ mode. This entire paper is based on simulation of DVB-T

\section{ACKNOWLEDGMENT}

We would like to thank Ms Drakshayini m n, our guide, and Mr Arun vikas singh, our HOD, and our other teachesr for helping us in doing this paper.

\section{REFERENCE:}

[1]. Zhengdao Wang," single carrier block transmission for OFDM" IEEE Transaction on Communication, March 2004, vol.52, issue 3, pp. 480-394.

[2]. L. Dai, Z. Wang, and Z. Yang, "Time-Frequency OFDM with High Spectral Efficiency and Performance in High Speed Environments, "IEEE Journal in Communications, vol. 30, no.4, 2012.

[3]. Banelli. P, Cacopardi. S, "Theoretical analysis and performance of OFDM signals in nonlinear AWGN NOISY CHANNEL", IEEE Tran. Commun., vol. 48, pp. 430-441, August 2002

[4]. B. R. Ballal; A Chadha; N Satam, "Orthogonal Frequency Division Multiplexing and its Applications and advantages", International Journal of Science and Research (IJSR), India, ISSN: 2319-7945.

\section{BIGRAPHIES}

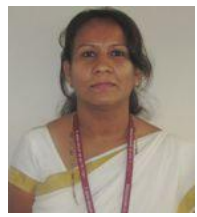

Ms. Drakshayini M NBe,Mtech(Phd) is an Associate Professor ofElectronics and Communication Engineering, T-John Institute of Technology, Bengaluru.

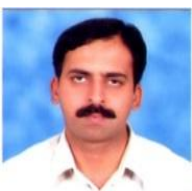

Dr. Arun Vikas Singh is the Head of the Department of Electronics and Communication Engineering, T-John Institute of Technology, Bengaluru.

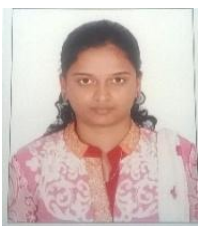

Ms. Vyshanava Nandini $\mathbf{S}$ is pursuing $\mathrm{M}$ tech degree in Digital Communication and Networking from T-John Institute of Technology, Bengaluru affliated to Visvesvaraya Technological University 